Trauma Berufskrankh 2016 · 18 (Suppl 5):S434-S443 DOI 10.1007/s10039-016-0166-1

Online publiziert: 30 . Juni 2016

๑) Springer-Verlag Berlin Heidelberg 2016

CrossMark

\title{
Helmut Maxeiner
}

Bereich Verbindungsstelle, Deutsche Gesetzliche Unfallversicherung, Berlin, Deutschland

\section{Sachleistungsaushilfe}

\section{Was ist zu beachten, wenn eine im Ausland versicherte Person in Deutschland einen Arbeitsunfall erleidet?}

und in der Schweiz ${ }^{4}$ gilt, haben auch die Staatsangehörigen dieser Staaten sowie in diesen Staaten versicherte Flüchtlinge und Staatenlose den gleichen Anspruch. Schließlich enthält eine Reihe bilateraler Abkommen über soziale Sicherheit, welche die Bundesrepublik Deutschland mit anderen Staaten geschlossen hat und in die die gesetzliche Unfallversicherung einbezogen ist, für Arbeitsunfälle und Berufskrankheiten dem VO-Recht vergleichbare Regelungen zur aushilfsweisen medizinischen Versorgung.

Personen aus dem vertragslosen Ausland haben hingegen keinen Anspruch auf aushilfsweise medizinische Versorgung, da Regelungen, die einen solchen Anspruch begründen würden, fehlen.

Die nachstehende Tabelle gibt einen Überblick zu der Anzahl der in Deutschland in der Zeit von 2006 bis 2015 aufgetretenen Fälle der Sachleistungsaushilfe und deren Verteilung auf die Bereider sozialen Sicherheit, Abl. L 166 vom 30. April 2004, S. 1-123, in der berichtigten Fassung des Abl. L 200 vom 07. Juni 2004 und in der Fassung der Verordnung (EG) Nr. 988/2009 des Europäischen Parlaments und des Rates vom 16. September 2009 zur Änderung der Verordnung Nr. 883/2004, Abl. L 284 vom 30. Oktober 2009, S.43-72.

2 Verordnung (EG) Nr. 987/2009 des Europäischen Parlaments und des Rates vom 16. September 2009 zur Festlegung der Modalitäten für die Durchführung der Verordnung (EG) Nr. 883/2004 über die Koordinierung der Systeme der sozialen Sicherheit, Abl. L 284 vom 30. Oktober 2009, S. 1-42.

3 Im Verhältnis zu den EWR-Staaten gelten die Verordnungen (EG) Nr. 883/2004 und Nr. 987/2009 seit dem 1. Juni 2012 durch den Beschluss des Gemeinsamen EWR-Ausschusses Nr. 76/2011 vom 1. Juli 2011 zur Änderung von Anhang VI (Soziale Sicherheit) und von Protokoll 37 zum EWR-Abkommen, Abl. L 262 vom 6. Oktober 2011, S.33-43.

\footnotetext{
${ }^{4}$ Im Verhältnis zur Schweiz gelten die VOen (EG) Nr. 883/2004 http://www.juris. de/jportal/portal/t/e0f/page/jurisw.psml? pid=Dokumentanzeige $\% 26$ showdoccase $=1$ \%26js_peid=Trefferliste\%26fromdoctodoc=yes \%26doc.id=jcg-32004R0883\%26doc.part=C $\% 26$ doc.price $=0.0 \% 23$ focuspoint und Nr. 987/2009 seit dem 1. April 2012 durch den Beschluss Nr. 1/2012des Gemischten Ausschusses, eingesetzt im Rahmen des Abkommens zwischen der Europäischen Gemeinschaft und ihren Mitgliedstaaten einerseits und der Schweizerischen Eidgenossenschaft andererseits über die Freizügigkeit vom 31. März 2012 zurErsetzung des Anhangs Il dieses Abkommens über die Koordinierung der Systeme der sozialen Sicherheit, Abl. L 262 vom 6. Oktober 2011, S.33-43.
}

che EU/EWR/Schweiz einerseits und Abkommenstaaten andererseits (• Abb. 1).

\section{Was umfasst der Anspruch auf Sachleistungsaushilfe?}

Nach den maßgeblichen Rechtsvorschriften des Art. 36 Verordnung (EG) Nr. 883/2004 und entsprechender Regelungen in Abkommen über soziale Sicherheit, z. B. Art. 23 des Abkommens mit Mazedonien ${ }^{5}$, haben betroffene Personen bei Versorgung in Deutschland Anspruch auf alle Sachleistungen nach dem Sozialgesetzbuch (SGB) VII, so als seien sie in Deutschland versichert. Dies gilt unabhängig davon, ob die gleiche Versorgung mit Sachleistungen in den Staaten, in denen die betroffenen Personen versichert sind, günstiger oder teurer ist und ob der Umfang des in diesen Staaten jeweils zur Verfügung stehenden Leistungspakets dem des SGB VII entspricht oder nicht.

In erster Linie geht es um die ambulante und stationäre medizinische Versorgung sowie alle weiteren damit in engerem Zusammenhang stehenden Maßnahmen wie physiotherapeutische Behandlungen, Versorgung mit Medikamenten, Hilfsmitteln kleinerer und größerer Art, Körperersatzstücken etc. $\mathrm{Zu}$ erbringen sind aber auch besondere Sachleistungen, die in den Leistungskatalogen anderer Staaten nicht enthalten sind, wie Berufshilfemaßnahmen, Kraft-

\footnotetext{
${ }^{5}$ Abkommen zwischen der Regierung der Bundesrepublik Deutschland und der mazedonischen Regierung über Soziale Sicherheit vom 8. Juli 2003, BGBIII 2004, S. 1068.
} 


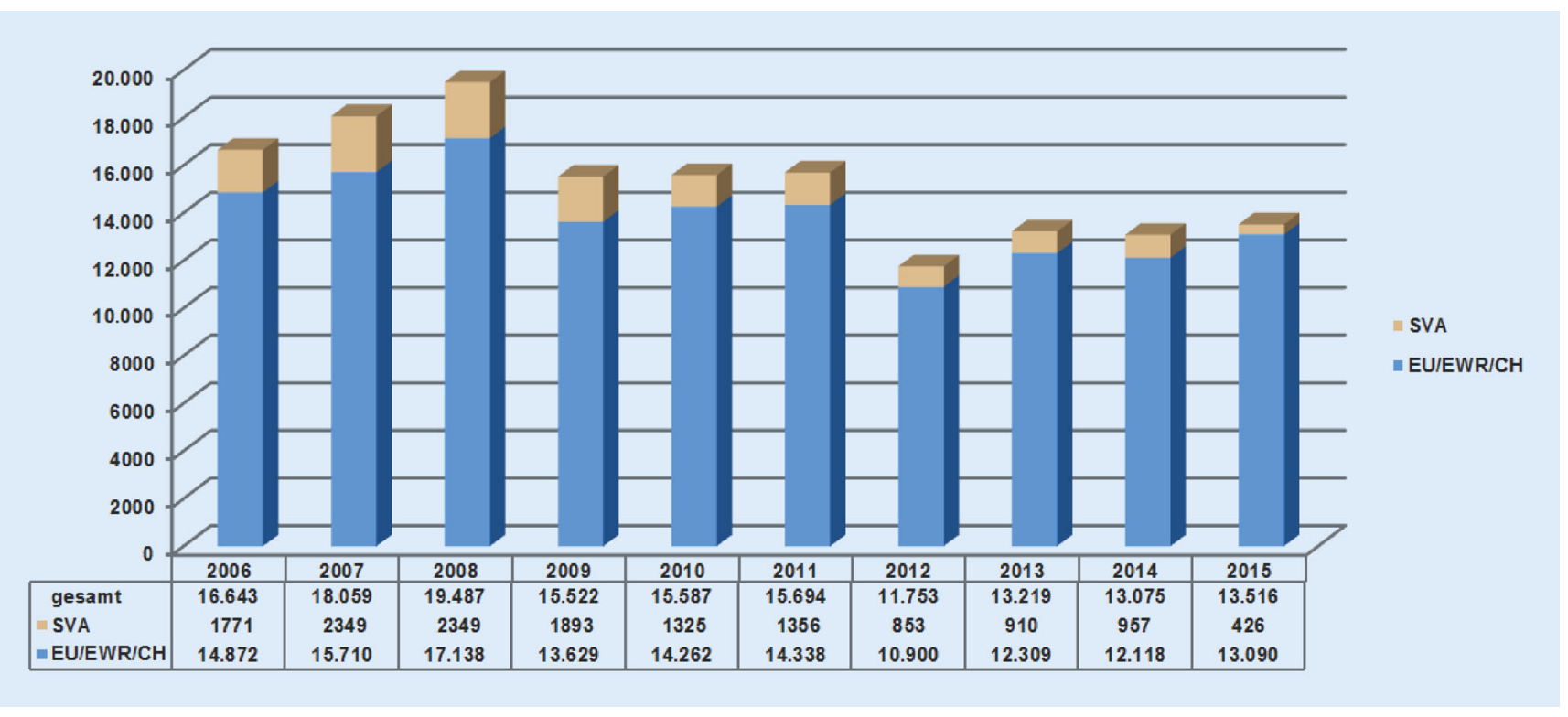

Abb. 1 ॥ Überblick der Sachleistungsaushilfefälle. SVA Sozialversicherungsabkommen, EU/EWR/CH Europäische Union/ Europäischer Wirtschaftsraum/Schweiz

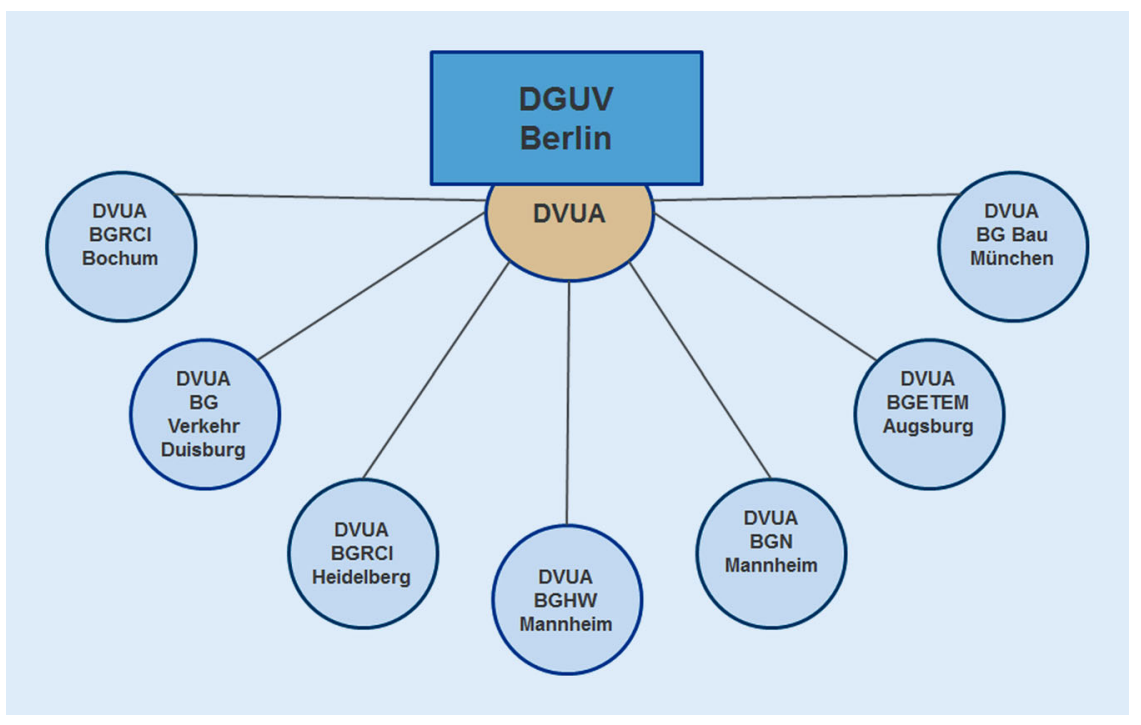

Abb. $2 \Delta$ Organisation der Deutschen Verbindungsstelle Unfallversicherung - Ausland (DVUA)

fahrzeughilfe und Wohnungshilfe. Bei diesen besonderen Sachleistungen ergeben sich nahezu regelmäßig Probleme mit den zuständigen ausländischen Trägern, da diese die Berechtigung zur Erbringung dieser Leistungen zu ihren Lasten ablehnen. Einigungen werden oft erst nach langwierigen Auseinandersetzungen erzielt.

lage des $₫ 139$ a SGB VII unter anderem die Durchführung der Sachleistungsaushilfe übertragen worden.

\section{Organisation der DVUA}

Die DVUA ist in die Deutsche Gesetzliche Unfallversicherung Spitzenverband e. V. integriert. Seit Oktober 2014 hat sie ihren Standort in Berlin. Zuvor war sie in Sankt Augustin angesiedelt. Am Standort in Berlin wird die Grundsatzarbeit geleistet. Daneben werden die Einzelfälle im Verhältnis zu Malta, der kanadischen Provinz Québec und Zypern (griechischsprachiger Bereich) erledigt. An weiteren sieben Standorten bei sechs gewerblichen Berufsgenossenschaften erfolgt die Bearbeitung der Einzelfälle im Verhältnis zu 39 Staaten (• Abb. 2).

\section{Wer ist bei der Erbringung der Sachleistungsaushilfe beteiligt?}

Da sich der Anspruch auf die aushilfsweise Versorgung auf alle Sachleistungen, die das SGB VII vorhält, erstreckt, sind auch alle Erbringer von Sachleistungen wie Ärzte - insbesondere Durchgangsärzte -, Krankenhäuser, Physiotherapeuten, Apotheken, Sanitätshäuser etc. auf der Leistungsseite beteiligt.

Auf administrativer Seite ist der Deutschen Verbindungsstelle Unfallversicherung - Ausland (DVUA) auf der Grund-

\section{Welche hauptsächlichen} Verpflichtungen bestehen für die Leistungserbringer und die DVUA?

Die betroffenen Personen haben die gleichen Ansprüche auf Sachleistungen, wie die in Deutschland versicherten Personen. Die Leistungserbringer haben daher die im Ausland versicherten Personen mit Anspruch auf Sachleistungsaushilfe in gleicher Weise zu versorgen, wie sie in der deutschen gesetzlichen Unfallversi- 
cherung versicherte Personen versorgen. Das bedeutet, die medizinische Rehabilitation ist mit allen geeigneten Mitteln zu betreiben. Daneben sind auch die für Inlandsfälle vorgesehenen Verfahren $\mathrm{zu}$ beachten. Deshalb ist der DVUA über den Beginn und den Verlauf der Behandlung wie in Inlandsfällen mittels Durchgangsarzt-, Nachschauberichten etc. zu berichten. Die DVUA hat das Heilverfahren wie in Inlandsfällen zu überwachen und zu steuern damit eine optimale Rehabilitation gewährleistet ist. Ergibt sich aus medizinischer Sicht die Notwendigkeit einer Weiterbehandlung bei einem Fachspezialisten, z. B. einem Handchirurgen, oder der Verlegung in eine andere Klinik, sind entsprechende Maßnahmen in Abstimmung mit der DVUA einzuleiten.

Die erbrachten Leistungen sind nach den mit der gesetzlichen Unfallversicherung für Inlandsfälle vereinbarten $\mathrm{Ge}$ bühren abzurechnen. Die DVUA rechnet anschließend die von ihr verauslagten Kosten in regelmäßigen Abständen mit den zuständigen ausländischen Trägern ab.

\section{Welche Voraussetzungen müssen erfüllt sein, dass ein Anspruch auf aushilfsweise Sachleistungen besteht?}

Nur wenn die betroffenen Personen in einem EU-Staat, einem EWR-Staat, der Schweiz oder in einem Abkommenstaat dem Schutz der gesetzlichen Unfallversicherung unterstehen und sie durch Dokumente, die eigens dazu geschaffen wurden, einen vorläufigen oder endgültigen Anspruch auf die aushilfsweise Versorgung mit Sachleistungen nachweisen können, ist die DVUA gleichermaßen berechtigt und verpflichtet, diesen sicherzustellen. Die betroffenen Abkommenstaaten können dem „Informationsblatt Sachleistungsaushilfe" der DVUA unter http:// publikationen.dguv.de/dguv/udt_dguv_ main.aspx?FDOCUID=24568 (abgerufen am 8.4.2016) entnommen werden.

Den Versicherungsschutz weisen die betroffenen Personen im EU-Bereich durch die Versicherungsbescheinigung A1 (• Abb. 3 ) oder E 101, im

Trauma Berufskrankh 2016 · 18 (Suppl 5):S434-S443 DOI 10.1007/s10039-016-0166-1

(c) Springer-Verlag Berlin Heidelberg 2016

\section{H. Maxeiner}

\section{Sachleistungsaushilfe. Was ist zu beachten, wenn eine im Ausland versicherte Person in Deutschland einen Arbeitsunfall erleidet?}

\section{Zusammenfassung}

In EU(Europäische Union)-/EWR(Europäischer Wirtschaftsraum)-Staaten, der Schweiz und in bestimmten Abkommenstaaten versicherte Personen haben bei Arbeitsunfall/Berufskrankheit während des vorübergehenden oder gewöhnlichen Aufenthalts in Deutschland Anspruch auf aushilfsweise Versorgung mit Sachleistungen (Sachleistungsaushilfe). Der Anspruch umfasst alle Sachleistungen nach dem SGB VII. Auf der Leistungsseite sind alle Leistungserbringer beteiligt, auf der administrativen Seite die Deutsche Verbindungsstelle Unfallversicherung - Ausland (DVUA). Zum Nachweis der Versicherung und des Anspruchs auf aushilfsweise Versorgung müssen die betroffenen Personen bestimmte Bescheinigungen vorlegen. Führen sie diese nicht mit sich, ist es wichtig, zu Beginn der Versorgung seitens der Leistungserbringer von den betroffenen Personen Informationen zu erfragen, welche die DVUA in die Lage versetzen, nachträglich zu prüfen, ob ein Anspruch auf aushilfsweise Versorgung besteht. Die entstandenen Kosten rechnet die DVUA nach Erstattung gegenüber den Leistungserbringern mit den zuständigen ausländischen Trägern ab.

\section{Schlüsselwörter}

Arbeitsunfall · Berufskrankheit · EURecht · Deutsche Verbindungsstelle Unfallversicherung - Ausland (DVUA) . Kostenerstattung

\section{Assistance with benefits in kind. What must be considered when a person insured in another country suffers an accident at work in Germany?}

\section{Abstract}

Individuals from member states of the European Union (EU), the European Economic Area (EEA), Switzerland and certain states which have social security agreements with Germany, who are insured against accidents at work and occupational diseases have the right to temporary benefits in kind during a temporary stay or permanent residence in Germany. The rights include all benefits in kind on the basis of the German social security statutes (Sozialgesetzbuch, SGB VII). Involved on the side of providing benefits in kind are all persons and institutions, which have to assure the benefits in kind and on the administrative side the German coordination office for foreign accident insurance (Deutsche Verbindungsstelle Unfallversicherung Ausland, DVUA). To confirm that the person is entitled to insurance and the right of benefits in kind, the person concerned has to present certain certificates. If these cannot be presented it is important at the beginning of providing benefits in kind to provide information that gives the DVUA the possibility to retrospectively check whether the right of benefits in kind exists. The DVUA pays the incidental costs of the benefits in kind and afterwards sends a claim for reimbursement to the appropriate foreign institution.

\section{Keywords}

Accident at work - Occupational disease . European Union - German administrative coordination - Reimbursement of costs
Abkommensbereich beispielsweise im Verhältnis zu Serbien ${ }^{6}$ durch die Be-

\footnotetext{
${ }^{6}$ Es gilt das Abkommen zwischen der Bundesrepublik Deutschland und der Sozialistischen Föderativen Republik Jugoslawien über Soziale Sicherheit vom 12. Oktober 1968, BGBI II 1969, 1438 , i.d.F. des Änderungsabkommens vom 30. September 1974, BGBI II 1975, 390, weiter.
}

scheinigung DE 101 SRB (• Abb. 4) nach.

Grundsätzlich können betroffene Personen aus EU-, EWR-Staaten und der Schweiz vorläufig mit Sachleistungen der Unfallversicherung versorgt werden, wenn sie zu Beginn der Behandlung eine gültige Versicherungsbescheinigung A1 oder E 101 sowie eine Europäische Krankenversicherungskarte (EHIC), eine pro- 


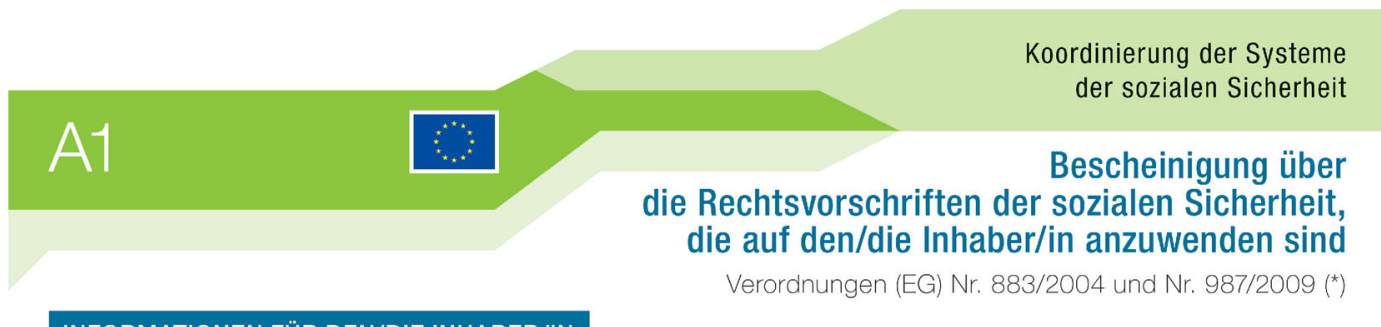

\section{INFORMATIONEN FÜR DEN/DIE INHABER/IN}

Dieses Dokument dient als Bescheinigung über die Sozialversicherungsvorschriften, die für Sie gelten, und als Bestätigung, dass Sie in einem anderen Staat keine Beiträge zu zahlen haben.

Bevor Sie den Staat, in dem Sie versichert sind, verlassen, um in einem anderen Staat eine Arbeit aufzunehmen, sollten Sie sicherstellen, dass Sie über die Dokumente verfügen, die Sie berechtigen, die notwendigen

Sachleistungen (medizinische Versorgung, stationäre Behandlung usw.) im Staat Ihrer Erwerbstätigkeit zu erhalten.

- Wenn Sie sich im Staat Ihrer Erwerbstätigkeit vorübergehend aufhalten, beantragen Sie bei Ihrem

Krankenversicherungsträger eine Europäische Krankenversicherungskarte (EKVK/EHIC). Sie müssen diese

Karte bei Ihrem Gesundheitsdienstleister vorlegen, wenn Sie während Ihres Aufenthalts Sachleistungen in

Anspruch nehmen müssen.

- Wenn Sie sich im Staat Ihrer Erwerbstätigkeit niederlassen, beantragen Sie bei Ihrem

Krankenversicherungsträger das Formular S1 und übermitteln dieses schnellstmöglich dem zuständigen

Krankenversicherungsträger des Ortes, an dem Sie Ihre Erwerbstätigkeit ausüben $\left(^{* *}\right)$.

Der Versicherungsträger im Aufenthaltsstaat wird bei einem Arbeitsunfall oder einer Berufskrankheit vorläufig besondere Leistungen erbringen.

\section{ANGABEN ZUR PERSON DES INHABERS/DER INHABERIN}

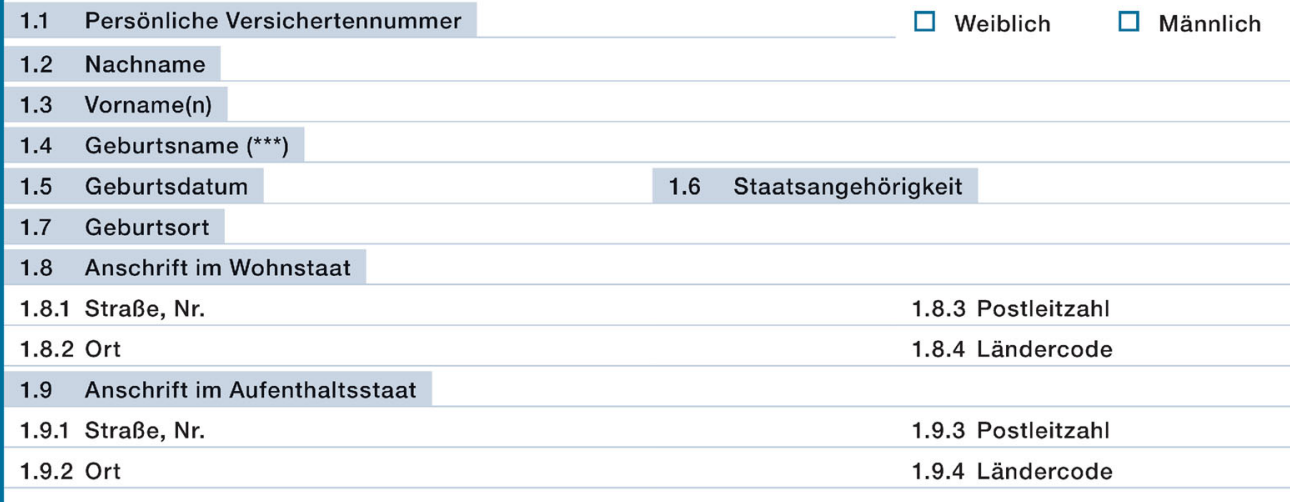

\section{MITGLIEDSTAAT, DESSEN RECHTSVORSCHRIFTEN ANZUWENDEN SIND}
2.1 Mitgliedstaat
2.2 Anfangsdatum
$2.3 \quad$ Enddatum
$\square$ 2.4 Die Bescheinigung gilt für die Dauer der Tätigkeit
$\square 2.5$ Die Feststellung ist vorläufig
$\square 2.6$ Übergangsbestimmungen finden Anwendung gemäß Verordnung (EG) Nr. 883/2004

(*) Verordnung (EG) Nr. 883/2004, Artikel 11 bis 16, und Verordnung (EG) Nr. 987/2009, Artikel 19.

$\left({ }^{*}\right)$ In Spanien muss das entsprechende Dokument der Provinzialdirektion der staatlichen Sozialversicherungsanstalt (INSS) des Wohnorts und in Schweden sowie Portugal dem jeweiligen Sozialversicherungsträger des Wohnorts übermittelt werden.

$\left.{ }^{(\star \star}\right)$ Liegen dem Träger hierzu keine Angaben vor, informiert der/die Inhaber/in diesen entsprechend.

Abb. $3<$ Versicherungsbescheinigung $A 1$ (auszugsweise)

CEuropäische Kommission 


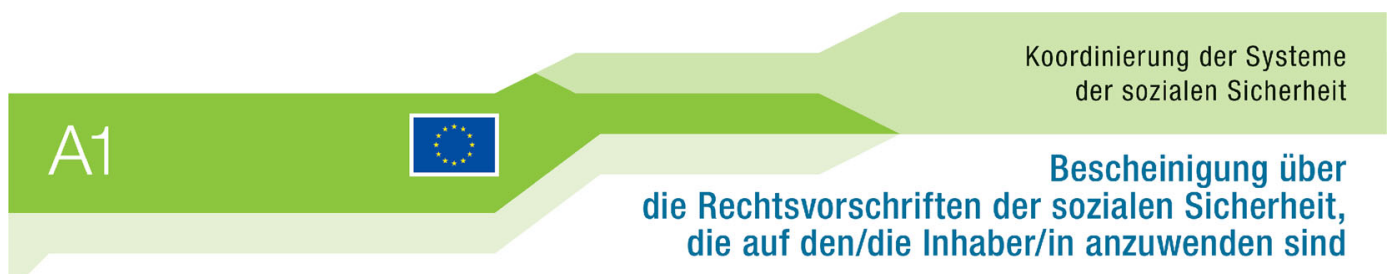

\section{STATUSBESTÄTIGUNG}

3.1 Entsandte/r Arbeitnehmer/in

3.3 Entsandte selbständig erwerbstätige Person

$\square \quad 3.5$ Beamter/Beamtin

$\square$ 3.7 Zum Kreis der Seeleute gehörig

$\square \quad 3.9$ In einem Staat als Beamter/Beamtin und in einem anderen Staat oder mehreren anderen Staaten als beschäftigte/selbstständig erwerbstätige Person tätig
3.2 Arbeitnehmer/in arbeitet in zwei oder mehr Staaten

$\square$ 3.4 Selbstständige/r, die/der in zwei oder mehr Staaten erwerbstätig ist

$\square \quad 3.6$ Vertragsbedienstete

$\square \quad 3.8$ In verschiedenen Staaten als beschäftigte und selbstständig erwerbstätige Person tätig

$\square \quad 3.10$ Mitglied von Flug- oder Kabinenbesatzung

$\square$ 3.11 Ausnahmevereinbarung

\section{ANGABEN ZUM ARBEITGEBER/ZUR SELBSTSTÄNDIGEN ERWERBSTÄTIGKEIT}

$\square$ 4.1.1 Arbeitnehmer/-in

$\square$ 4.1.2 Selbstständig erwerbstätig

4.2 Kenn-Nummer des Arbeitgebers/der selbstständigen Erwerbstätigkeit

4.3 Name oder Firmenbezeichnung

4.4 Ständige Anschrift

4.4.1 Straße, $\mathrm{Nr}$

4.4.2 Ländercode

4.4.3 Ort

4.4.4 Postleitzahl

5. ANGABEN ZUM ARBEITGEBER/ZUR SELBSTSTÄNDIGEN ERWERBSTÄTIGKEIT AN DEM ORT, AN DEM EINE ERWERBSTÄTIGKEIT AUSGEÜBT WIRD

5.1 Name(n) oder Firmenname(n) und Kennnummer(n) des Betriebs/der Betriebe bzw. des Schiffs/der Schiffe oder der Heimatbasis/der Heimatbasen, wo Sie beschäftigt sein werden

5.2 Anschrift(en) oder Name(n) des Schiffs/der Schiffe oder der Heimatbasis/der Heimatbasen, wo Sie im/in den „Aufnahme“-Staat/en (selbstständig) erwerbstätig sein werden

Abb. $3 \triangleleft$ Versicherungsbescheinigung $A 1$ (auszugsweise) (Fortsetzung) 


\section{СПОРАЗУМ ИЗМЕЪУ СОЦИЈАЛИСТИЧКЕ ФЕДЕРАТИВНЕ РЕПУБЛИКЕ ЈУГОСЛАВИЈЕ И САВЕЗНЕ РЕПУБЛИКЕ НЕМАЧКЕ О СОЦИЈАЛНОМ ОБЕЗБЕЪЕЊУ \\ ABKOMMEN ZWISCHEN DER SOZIALISTISCHEN FÖDERATIVEN REPUBLIK JUGOSLAWIEN UND DER BUNDESREPUBLIK DEUTSCHLAND ÜBER SOZIALE SICHERHEIT}

\begin{tabular}{|c|c|c|c|}
\hline $\begin{array}{l}\text { Потврда о примени српских пра } \\
\text { запошљавања у Немачкој }{ }^{1)} \\
\text { Чпан }\end{array}$ & прописа приликом & $\begin{array}{l}\text { inigung über d } \\
\text { vorschriften bei }\end{array}$ & $\begin{array}{l}\text { Anwendung der serbischen } \\
\text { chäftigung in Deutschland }{ }^{1)}\end{array}$ \\
\hline$\square$ Запослени / Arbeitnehmer & $\square$ Друго лице & Person & \\
\hline $\begin{array}{l}\text { Презиме / } \\
\text { Name }\end{array}$ & $\begin{array}{l}\text { Име / } \\
\text { Vorname }\end{array}$ & $\begin{array}{l}\text { Датум рођења / } \\
\text { Geburtsdatum }\end{array}$ & $\begin{array}{l}\text { Број осигураника (ЈМБГ) / } \\
\text { Versichertennummer }\end{array}$ \\
\hline Aдpeca / Anschrift & & & \\
\hline
\end{tabular}

\section{2. Послодавац у Србији / Arbeitgeber in Serbien}

Назив или фрирма послодавца / Name oder Firma

Адреса / Anschrift

3. $\square$ у периоду од ......................................... лице наведено у пољу 1 .

$\square$ упућује се у предузеће у Немачкој, наведено у пољу 4.

$\square$ биће запослено у предузеће у Немачкој, наведено у пољу 4 / обављаће самосталну делатност.

$\square$ Лице наведено у пољу 1. је српски држављанин и запослен је од стране државних органа Републике Србије или шефа, члана или службеника дипломатске мисије или конзуларног представништва Републике Србије.
3. $\square$ Der in Feld 1 Genannte wird für die Zeit

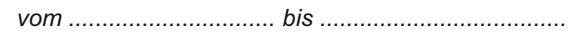

$\square \mathrm{zu}$ dem in Feld 4 genannten Unternehmen in Deutschland entsandt.

$\square$ bei dem in Feld 4 genannten Unternehmen in Deutschland beschäftigt/selbständig erwerbstätig sein.

$\square$ Der in Feld 1 Genannte ist serbischer Staatsangehöriger und wird von der Republik Serbien oder von dem Leiter, einem Mitglied oder einem Bediensteten einer amtlichen Vertretung der Republik Serbien beschäftigt.

4. Предузеће у Немачкој / Unternehmen in Deutschland

Назив или фрирма послодавца / Name oder Firma

Адреса / Anschrift

5. За лице наведено у пољу 1. важе, у складу са

$\square$ чланом 6. став 1 (упућивање)

$\square$ чланом 6. став 2 (запослење у транспортном предузећу)

$\square$ чланом 7. (запослење на поморском броду)

$\square$ чланом 9. (дипломатско и конзуларно представништво)

$\square$ чланом 10. (договор надлежних органа о изузетку)

Споразума

$$
\text { од / vom }
$$

до / bis

5. Für den in Feld 1 Genannten gelten nach

$\square$ Art. 6 Abs. 1 (Entsendung)

$\square$ Art. 6 Abs. 2 (Beschäftigung für ein Transportunternehmen)

$\square$ Art. 7 (Beschäftigung auf einem Seeschiff)

$\square$ Art. 9 (diplomatische und konsularische Vertretung)

$\square$ Art. 10 (Ausnahmevereinbarung)

des Abkommens

die serbischen Rechtsvorschriften über Soziale Sicherheit.

6. Носилац осигурања у Србији / Serbischer Träger

Назив / Bezeichnung

Матични број установе / Kenn-Nr.

Адреса / Anschrift

Печат / Stempel

Датум / Datum

Потпис / Unterschrift

Abb. $4<$ Versicherungsbescheinigung DE 101 SRB (auszugsweise) 


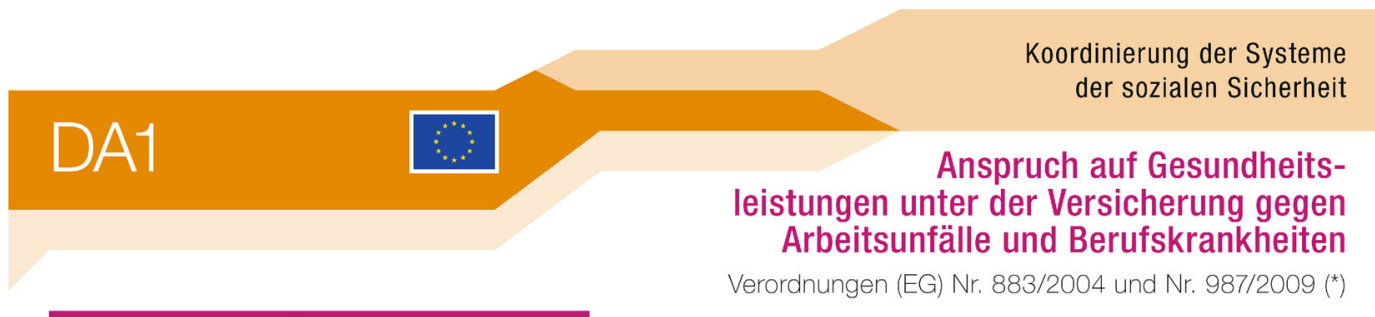

\section{INFORMATIONEN FÜR DEN/DIE INHABER/IN}

Dieses Dokument dient versicherten Personen, die sich in einen anderen EU-Staat, als den Staat der Versicherung gegen Arbeitsunfälle und Berufskrankheiten begeben, um dort zu wohnen oder sich aufzuhalten. Sie müssen dieses Dokument dem Krankenversicherungsträger/ dem Versicherungsträger für Arbeitsunfälle und Berufskrankheiten im Wohn- oder Aufenthaltsstaat vorlegen, um einen Anspruch auf Gesundheitsleistungen zu erwerben.

Unter Umständen haben Sie Anspruch auf eine zusätzliche Erstattung gemäß den am Aufenthaltsort geltenden nationalen Erstattungssätzen.

Nähere Informationen hierzu erhalten Sie von Ihrem Krankenversicherungsträger. Eine Liste mit

Krankenversicherungsträgern finden Sie unter: http://ec.europa.eu/social-security-directory/

\section{ANGABEN ZUR PERSON DES INHABERS/DER INHABERIN}

1.1 Persönliche Versichertennummer im zuständigen Mitgliedstaat

1.2 Nachname

1.3 Vorname(n)

1.4 Geburtsname (**)

1.5 Geburtsdatum

1.6 Status

$\square$ 1.6.1 Arbeitnehmer/in

1.6.2 Selbstständig erwerbstätige Person

1.6.3 Arbeitslos

1.7 Anschrift im Wohnsitz-/Aufenthaltsstaat
1.7.1 Straße, Nr.
1.7.3 Postleitzahl
1.7.2 Ort
1.7.4 Länderschlüssel UK

\section{DER/DIE INHABER/IN KANN SACHLEISTUNGEN IN ANSPRUCH NEHMEN}

$\square$ 2.1.1 wegen Arbeitsunfall $\square$ 2.1.2 wegen einer Berufskrankheit

2.2 Voraussichtlicher Zeitraum der Behandlung

$\square$ 2.2.1 für einen Zeitraum nach den Rechtsvorschriften des Wohnsitzstaats

$\square$ 2.2.2 Beginn

Ende

2.2.3 für höchstens drei Monate

2.2.4 für einen unbegrenzten Zeitraum

(*) Verordnung (EG) Nr. 883/2004 Artikel 36 und Verordnung (EG) Nr. 987/2009 Artikel 33.

$\left(^{\star *}\right)$ Liegen dem Träger hierzu keine Angaben vor, informiert der/die Inhaber/in diesen entsprechend.

(O) Europäische Kommission

Abb. $5<$ Anspruchsbescheinigung DA1 


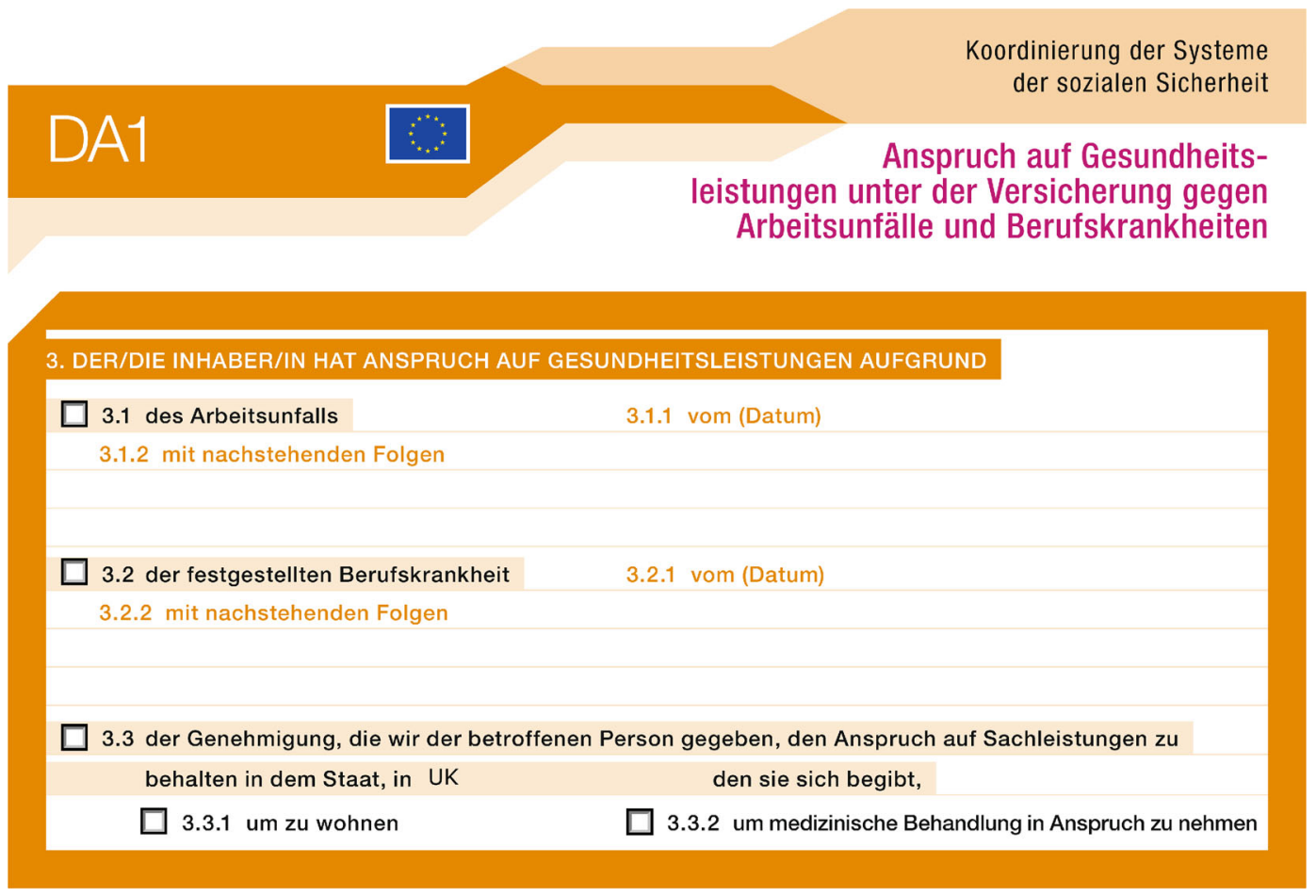

\section{DER BERICHT UNSERES BEHANDELNDEN ARZTES}

$\square 4.1$ ist in einem versiegelten Umschlag beigefügt $\square 4.2$ wird auf Anfrage zugeleitet

$\square 4.3$ wurde versendet
4.3.1 am
4.3.2 an

$\square 4.4$ wurde nicht erstellt

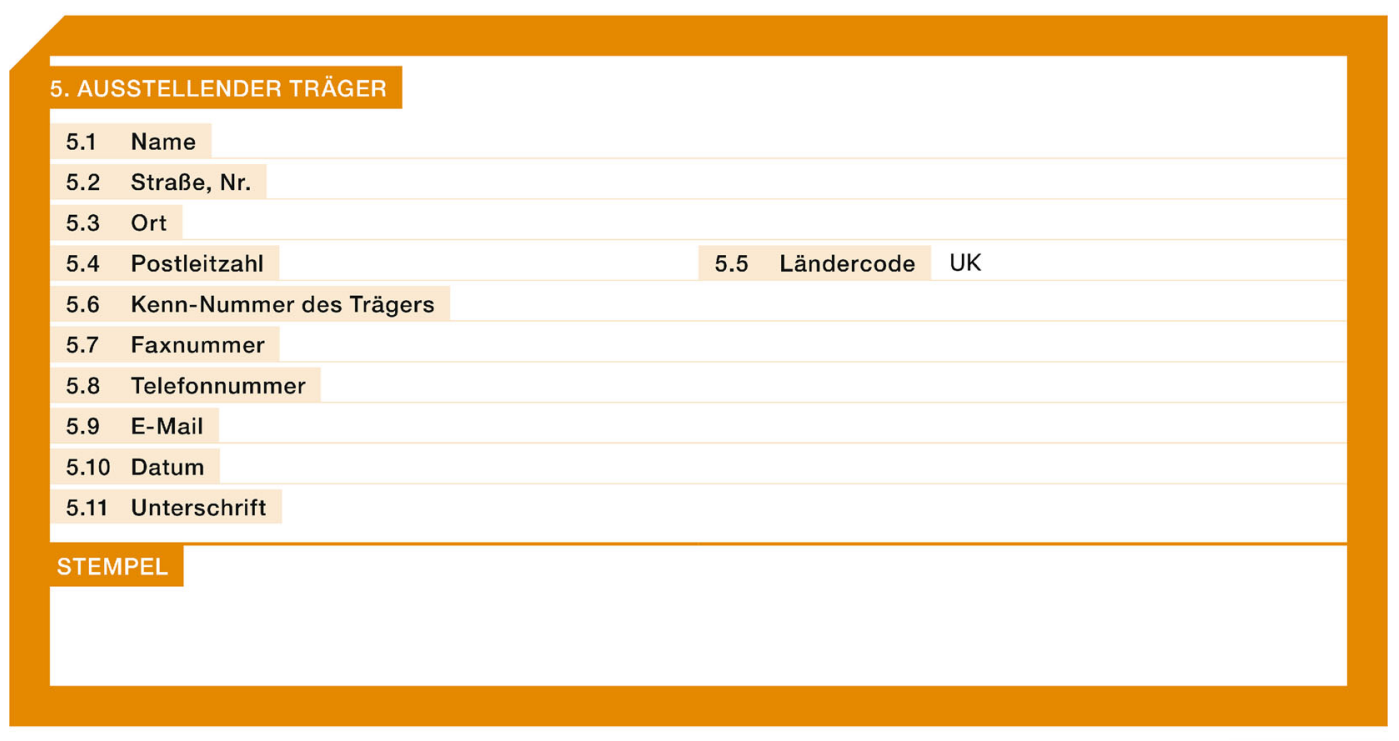

Abb. $5<$ Anspruchsbescheinigung DA1 (Fortsetzung) 
СПОРАЗУМ ИЗМЕЪУ СОЦИЈАЛИСТИЧКЕ ФЕДЕРАТИВНЕ РЕПУБЛИКЕ ЈУГОСЛАВИЈЕ

И САВЕЗНЕ РЕПУБЛИКЕ НЕМАЧКЕ О СОЦИЈАЛНОМ ОБЕЗБЕЪЕЊУ

ABKOMMEN ZWISCHEN DER SOZIALISTISCHEN FÖDERATIVEN REPUBLIK JUGOSLAWIEN UND DER BUNDESREPUBLIK DEUTSCHLAND ÜBER SOZIALE SICHERHEIT

Потврда оправу на давања унатури у случају повреде на раду или професионалне болести за време боравка I пребивалишта у Немачкој / Bescheinigung über Anspruch auf Sachleistungen bei Arbeitsunfall oder Berufskrankheit während des Aufenthalts in Deutschland

Чл. 4, 21. и 22. Споразума / Art. 4, Art. 21, Art. 22 des Abkommens

Чл. 4. и 9. Споразума органа за везу / Art. 4, Art. 9 der Vereinbarung der Verbindungsstellen

1 Осигураник/ Versicherte Person

1.1 Презиме / Name

Klicken Sie hier, um Text einzugeben

1.2 Име/ Vorname

Klicken Sie hier, um Text einzugeben.

1.3 Девојачко презиме / Geburtsname

Klicken Sie hier, um Text einzugeben.

1.4 Датум рођења/ Geburtsdatum

Klicken Sie hier, um Text einzugeben

1.5 Број осигураника у Србији (ЈМБГ) / Versichertennummer in

Klicken Sie hier, um Text einzugeben Serbien (JMBG)

1.6 Број документа помоћног немачког носиоца / Aktenzeichen des aushelfenden deutschen Trägers

1.7 Адреса у Србији/ Anschrift in Serbien ${ }^{1)}$

Klicken Sie hier, um Text einzugeben

Klicken Sie hier, um Text einzugeben

1.8 Адреса у Немачкој/ Anschrift in Deutschland ${ }^{11}$

$1.9 \square$ Повреда на раду од / Arbeitsunfall vom...Klicken Sie hier, um Text einzugeben.

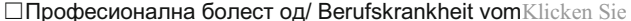
hier, um Text einzugeben.

\begin{tabular}{|c|c|c|c|}
\hline 2 & & & \\
\hline 2.1 & $\begin{array}{l}\square \text { Ваш образац SRB } 107 \text { DE одKlicken Sie hier, um Text einzu- } \\
\text { geben. }\end{array}$ & 2.1 & $\begin{array}{l}\square \text { Unter Bezugnahme auf Ihren Vordruck SRB } 107 \text { DE } \\
\text { vom Klicken Sie hier, um Text einzugeben. }\end{array}$ \\
\hline 2.2 & $\begin{array}{l}\square \text { У вези са боравком осигураника у Немачкој саопштавамо } \\
\text { следеће: } \\
\text { Klicken Sie hier, um Text einzugeben. }\end{array}$ & 2.2 & $\begin{array}{l}\square \text { Im Zusammenhang mit dem Aufenthalt der versi- } \\
\text { chertenPerson in Deutschland teilen wir Folgendes } \\
\text { mit: } \\
\text { Klicken Sie hier, um Text einzugeben. }\end{array}$ \\
\hline 2.3 & На основу & 2.3 & Aufgrund \\
\hline & $\begin{array}{l}\square \text { под } 1.9 \text { наведене повреде на раду/професионалне } \\
\text { болести са у даљем тексту наведеним последицама }{ }^{2)} \text { Kli- } \\
\text { ckenSiehier, umTexteinzugeben. }\end{array}$ & & $\begin{array}{l}\square \text { des unter } 1.9 \text { genannten Arbeitsunfalls/der Berufs- } \\
\text { krankheit mit nachstehend bezeichneten Folgen }{ }^{2)} \\
\text { Klicken Sie hier, um Text einzugeben. }\end{array}$ \\
\hline 2.4 & $\begin{array}{l}\square \text { наше сагласности да осигураник свој боравак или } \\
\text { пребивалиште премести у Немачку }\end{array}$ & 2.4 & $\begin{array}{l}\square \text { der der versicherten Person von uns hiermit erteilten } \\
\text { Zustimmung, ihren vorübergehenden oder gewöhnli- } \\
\text { chen Aufenthalt nach Deutschland zu verlegen }\end{array}$ \\
\hline & $\begin{array}{l}\text { Овом осигуранику се због последица повреде на раду / } \\
\text { професионалне болести могу пружити давања у натури: }\end{array}$ & & $\begin{array}{l}\text { können der versicherten Person wegen der Folgen } \\
\text { des Arbeitsunfalls/der Berufskrankheit Sachleistun- } \\
\text { gen erbracht werden }\end{array}$ \\
\hline 2.5 & $\begin{array}{l}\square \text { у времену одKlicken Sie hier, um Text einzugeben.доKlicken } \\
\text { Sie hier, um Text einzugeben. }\end{array}$ & 2.5 & $\begin{array}{l}\square \text { in der Zeit vom Klicken Sie hier, um Text einzugeben. } \\
\text { bis Klicken Sie hier, um Text einzugeben. }\end{array}$ \\
\hline 2.6 & $\begin{array}{l}\square \text { одKlicken Sie hier, um Text einzugeben.временски } \\
\text { неограничено. }\end{array}$ & 2.6 & $\begin{array}{l}\square \text { vom Klicken Sie hier, um Text einzugeben. an zeitlich } \\
\text { unbegrenzt. }\end{array}$ \\
\hline
\end{tabular}

Abb. $6 \triangleleft$ Anspruchsbescheinigung DE 123 SRB (auszugsweise) 
visorische Ersatzbescheinigung (PEB) oder die Bescheinigung E 106 vorweisen können. Gleiches gilt im Verhältnis zu den Abkommenstaaten Mazedonien und Serbien, wenn die Bescheinigungen D/RM 111, bzw. DE 111 SRB vorgelegt werden.

Eine endgültige Bestätigung des Anspruchs auf aushilfsweise Versorgung seitens des zuständigen ausländischen Trägers mit der Erklärung der Bereitschaft zur Übernahme der entstehenden Kosten erfolgt im EU-Bereich mit der von der betroffenen Person vorzulegenden Anspruchsbescheinigung DA1 (- Abb. 5) oder E 123, im Abkommensbereich beispielsweise im Verhältnis zu Serbien mit der Anspruchsbescheinigung DE 123 SRB (• Abb. 6).

Kann die betroffene Person keine Bescheinigung(en) oder nur einen Teil der Bescheinigungen vorlegen, ist sie seitens der Leistungserbringer zu fragen, bei welchem ausländischen Träger sie unfallversichert ist. Falls ihr dieser nicht bekannt ist, muss unbedingt nach ihrer Staatsangehörigkeit, der Bezeichnung und genauen Anschrift ihres Arbeitgebers und ihres Herkunftsstaates gefragt werden. Hilfreich ist auch die Anfertigung einer Kopie vorhandener Personaldokumente, z. B. des Reisepasses. Die Angaben/Kopie sind der DVUA zu übermitteln, damit sie den zuständigen ausländischen Träger ermitteln und mit ihm in Kontakt treten kann.

\section{Problem des Time-Lag}

Hat die betroffene Person beim Leistungserbringer keine Anspruchsbescheinigung vorgelegt, benachrichtigt die DVUA den zuständigen ausländischen Träger unverzüglich, nachdem sie Kenntnis von einem möglichen Arbeitsunfall/ einer Berufskrankheit erlangt hat, und bittet, den Anspruch auf aushilfsweise Versorgung mit Sachleistungen zu prüfen und eine Anspruchsbescheinigung zu übermitteln. Im Gegensatz zur gesetzlichen Krankenversicherung reicht es im Bereich der gesetzlichen Unfallversicherung allein nicht aus, versichert $\mathrm{zu}$ sein, damit ein Leistungsanspruch verwirklicht werden kann. Es sind vielmehr vom zuständigen Träger zunächst die Kausalitätsfragen zu prüfen, und anschließend ist $\mathrm{zu}$ entscheiden, ob die Voraussetzungen eines Arbeitsunfalls/einer Berufskrankheit erfüllt sind. Bedauerlicherweise sind die Entscheidungsprozesse aufgrund der nationalen Gegebenheiten einiger Mitgliedstaaten der EU und einiger Abkommenstaaten sehr langwierig, weshalb in einer nicht geringen Zahl der Fälle der DVUA erst Wochen oder Monate nach Beginn der Versorgung Anspruchsbescheinigungen oder Ablehnungen zugehen.

Bis zum Eingang einer Anspruchsbescheinigung kann die DVUA den Leistungserbringern bedauerlicherweise keine Behandlungskosten erstatten, da sie keine Garantie der Kostenerstattung seitens des zuständigen ausländischen Trägers hat. Würde sie ohne Anspruchsbescheinigungen Erstattungen vornehmen, nähme sie ein nicht beherrschbares Kostenrisiko in Kauf. Die DVUA informiert die behandelnden Ärzte darüber im Einzelfall und bittet, die Information an andere Leistungserbringer, die von den Ärzten in das Rehabilitationsverfahren eingeschaltet wurden, wie Krankentransportunternehmen, Physiotherapeuten, Apotheken und Sanitätshäuser weiterzugegeben.

Ist die betroffene Person im ausländischen Staat krankenversichert, hat sie möglicherweise auf der Grundlage des EU-Rechts oder eines Abkommens über soziale Sicherheit einen Anspruch auf aushilfsweise medizinische Versorgung gegenüber der deutschen Krankenversicherung. Als Nachweis können die betroffenen Personen aus dem EU-, EWRBereich und der Schweiz die EHIC, eine PEB oder den Vordruck E 106 vorlegen, im Abkommensbereich z. B. im Verhältnis zu Serbien den Vordruck DE 111 SRB.

Besonders wichtig ist es in diesem $\mathrm{Zu}$ sammenhang, die betroffenen Personen aufzufordern, mit den Mustern 80 und 81 vorsorglich eine aushelfende deutsche Krankenkasse zu wählen. Wird später seitens des zuständigen ausländischen Trägers der Anspruch auf aushilfsweise Versorgung mit Sachleistungen wegen Arbeitsunfall/Berufskrankheit abgelehnt, was zur Folge hat, dass die DVUA keine Kosten erstatten kann, besteht für die Leistungserbringer die Möglichkeit, die erbrachten Leistungen mit der gewählten Krankenkasse abzurechnen. Ohne die Wahl einer aushelfenden Krankenkasse zu Beginn der Versorgung wird eine aushilfsweise Kostenerstattung seitens der Krankenversicherung möglicherweise nicht reibungslos verlaufen, da offen ist, welche Krankenkasse aushilfsweise die Kosten zu übernehmen hat.

\section{Informationen und Kontakte}

Insbesondere für Leistungserbringer hält die DVUA die folgenden Informationen bereit:

- „Informationsblatt Sachleistungsaushilfe"

http://publikationen.dguv.de/

dguv/udt_dguv_main.aspx?

FDOCUID $=24568$ (abgerufen

am 8.4.2016)

— „Schnellübersicht“

http://www.dguv.de/medien/inhalt/

internationales/pdf/schnell_bersicht.

pdf (abgerufen am 8.4.2016)

- Die Kontaktdaten der DVUA und ihrer Standorte können unter der folgenden Web-Adresse eingesehen werden:

http://www.dguv.de/de/Internationales/ Deutsche-Verbindungsstelle/

Ansprechpartner-und-Organisation/ index.jsp (abgerufen am 8.4.2016).

\section{Korrespondenzadresse}

\section{H. Maxeiner}

Bereich Verbindungsstelle, Deutsche Gesetzliche Unfallversicherung Glinkastr. 40, 10117 Berlin, Deutschland helmut.maxeiner@dguv.de

\section{Einhaltung ethischer Richtlinien}

Interessenkonflikt. H. Maxeiner gibt an, dass kein Interessenkonflikt besteht.

Dieser Beitrag beinhaltet keine vom Autor durchgeführten Studien an Menschen oder Tieren.

The supplement containing this article is not sponsored by industry. 